Check for updates

Cite this: RSC Adv., 2017, 7, 40411

Received 8th June 2017

Accepted 9th August 2017

DOI: 10.1039/c7ra06433k

rsc.li/rsc-advances

\section{Fish gelatin nanofibers prevent drug crystallization and enable ultrafast delivery}

\author{
Hyo Won Kwak, (DD a Heechang Woo, ${ }^{\text {I }}$ In-Chul Kim ${ }^{c}$ and Ki Hoon Lee ${ }^{* b d e}$
}

For drug delivery, uncontrolled drug crystallization is the most important and well-described problem which affects the compatibility between target drug and carrier polymer matrix. The crystallization of drugs makes a drug delivery system unstable and it causes a drop the drug loading efficiency and unpredictable release behavior. Recently, the electrospinning process which has rapid solvent evaporation speed has been proposed to prevent the drug crystallization during the drug loading process. In the present study, a fastdissolving nanofibrous drug delivery carrier was prepared via electrospinning of aqueous fish gelatin as the polymer carrier and caffeine as the hydrophilic model drug under environmentally friendly conditions. Fish gelatin electrospinning was able to produce caffeine loaded gelatin nanofibers with a diameter of 200$220 \mathrm{~nm}$. The X-ray diffraction (XRD) and differential scanning calorimetry (DSC) data clearly showed that the amorphous state of caffeine was well incorporated into ultrafine nanofibrous carrier whereas crystalline caffeine was dispersed into conventional fish gelatin film. Fish gelatin nanofibers were able to load two times more caffeine (20 $\mathrm{mg} \mathrm{m}^{-1}$ ) than conventional caffeine-loaded polymer nanofibers. The fish gelatin nanofibrous mat has good flexibility compared to film due to its interporous nanofiber network. The fish gelatin nanofibrous mats disintegrated within $1.5 \mathrm{~s}, 27$ times faster than films. Fish gelatin nanofibers also showed that most caffeine was released within 10 seconds, which is faster than fish gelatin film and free caffeine drugs. These fish gelatin nanofibers showed 10 times faster caffeine release rates than other caffeine loaded polymer nanofibers. Electrospun fish gelatin nanofibrous mats can be used in biomedical fields especially for the ultra fast delivery of hydrophilic drugs or active ingredients.

\section{Introduction}

Fast-disintegrating drug delivery systems (FDDS) have become an important and rapidly growing area in the pharmaceutical industry. ${ }^{1}$ Such FDDS carriers are easily wetted and disintegrated within $1 \mathrm{~min}$ on the tongue, releasing the drug or active ingredient instantaneously. ${ }^{2}$ This has many advantages, such as easy manufacturing, fast administration speed, accurate dosing, rapid onset of action and comfortable handling by patients. For this reason, many of the pharmaceutical companies have been tending to convert their drug products to fit into FDDS formulations. ${ }^{3}$

The selection of FDDS formulation is one of the most important and critical parameters to consider for the overall

\footnotetext{
${ }^{a}$ Department of Materials Science and Engineering, The University of Sheffield, Sheffield, S1 3JD, UK

${ }^{b}$ Department of Biosystems \& Biomaterials Science and Engineering, Seoul National University, Seoul 151-921, Republic of Korea. E-mail: prolee@snu.ac.kr

${ }^{c}$ Research Center for Biobased Chemistry, Korea Research Institute of Chemical Technology, Daejeon 34114, Republic of Korea

${ }^{d}$ Research Institute of Agriculture and Life Sciences, Seoul National University, Seoul 151-921, Republic of Korea

${ }^{e}$ Center for Food \& Bioconvergence, Seoul National University, Seoul 151-921, Republic of Korea
}

properties of a fast drug delivery carrier. The most common formula of FDDS are tablets and films and the latter film FDDS carriers are more preferred to tablets. ${ }^{4}$ Because the tablets have certain disadvantages as difficult to carry, store, handle, and slow drug release behavior due to less surface area. ${ }^{5}$ However, film types of FDDS carrier also have a problem that needs the additional components such as plasticizer which could be flexible for storage and handling. ${ }^{6}$ Furthermore, it could induce unexpected drug crystallization during film forming process. ${ }^{7}$ Crystallization of drug makes the drug delivery system unstable and it causes dropping the drug loading efficiency and slow release behavior. ${ }^{8}$

Recently, electrospinning process was introduced to overcome the disadvantage of tablets and film based FDDS carrier. ${ }^{9}$ This process is possible to fabricate the submicrometer (micro to nano) diameter polymeric fibers using electric fields driven polymer solution jet. ${ }^{10}$ The extraordinary higher surface area and porosity of nanofibrous structure can promote the wetting by salivary substances and facilitate the rapid drugs or active ingredients release. ${ }^{11}$ Also, the fast solvent evaporation speed prevents the formation and growth of drug crystal compared to conventional solvent evaporation film casting method. ${ }^{\mathbf{9 2}, \mathbf{1 3}}$

Nowadays, the various synthetic polymers have been introduced for electrospun nanofibrous FDDS carrier. Polyvinyl alcohol (PVA), polycaprolactone (PCL), and polyvinylpyrrolidone 
(PVP) were fabricated into nanofibrous FDDS carrier using elec-

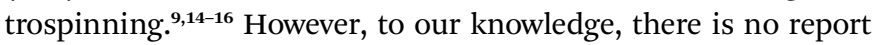
in terms of natural polymer; especially food based FDDS nanofibrous carrier using water as a solvent so far. Natural polymers are candidate materials to be developed as drug delivery carrier matrix. Because these polymers are naturally occurring and possess desirable properties in terms of biocompatibility and biodegradability. Furthermore, the use of water as a solvent has the advantage of being more economical and safer than organic solvent systems.

Gelatin is a typical hydrophilic natural protein polymer which obtained by partial hydrolysis and thermal degradation of various collagen sources from the beef, pork, and fish food processing. ${ }^{17-19}$ Among these, gelatin from marine sources such as fish skin or bone has gained an attention as a base material in the biomedical scaffold, food packaging, and pharmaceutical industry due to its low cost and multi-functionality. It is suitable for the preparation of solid dispersions by the solvent method due to their good solubility in water, rapid water uptake. It also has the ability to maintain the solution state at room temperature, while mammal gelatin easily converts into gel state which is difficult to fabricate to film or fibrous form. ${ }^{20}$ Furthermore, from a religious point of view, fish gelatin can be used extensively. ${ }^{21}$ For example, Muslims and Hindus prohibit consuming the porcine and bovine product, respectively. Taking all of these reasons together, the use of fish gelatin has the potential to become increasingly widespread.

Previously, we fabricated the electrospun nanofibrous fish gelatin tissue engineering scaffold and found that the gelatin from cold water fish was easily fabricated under the green process condition. ${ }^{22}$ This process uses water as a solvent at room temperature, without any organic solvent, polymer additives, and temperature control facilities.

In the present study, we fabricated the nanofiber-based FDDS carrier using the electrospinning with the hydrophilic aqueous fish gelatin as a matrix and caffeine which has high crystallization tendency as a hydrophilic model drug. Effect of electrospinning process on the caffeine crystallization was investigated using optical and electron microscopy, differential scanning calorimetry (DSC), X-ray diffraction (XRD). The dissolution and wetting behavior of fish gelatin nanofibrous FDDS carrier were also investigated. Finally, caffeine release behavior was explored.

\section{Experimental}

\subsection{Materials}

Cold-water fish gelatin (FG) and caffeine (CAF) were purchased from Sigma Aldrich (Yongin, Korea). All chemicals were of analytical reagent grade and used without further purification.

\subsection{Preparation and characterization of caffeine loaded fish gelatin solutions}

FG was dissolved in distilled water $(40 \%, \mathrm{w} / \mathrm{v})$ at room temperature for $1 \mathrm{~h}$ with vigorous stirring. Various concentration of CAF $(0,0.4,0.8,1.2,1.6$ and $2.0 \%$, w/v) was mixed with FG solutions. After complete dissolution of CAF, CAF-FG solution was filtered with filter paper for insoluble impurity removal and stored at room temperature before being used. The viscosity of the dope solution was analysed with a DV-E viscometer (Brookfield, Middleboro, MA, USA) in a temperature controlled chamber $\left(25^{\circ} \mathrm{C}\right)$ and the conductivity was measured using a SG23-SevenGo Duo ${ }^{\mathrm{TM}} \mathrm{pH} /$ conductivity (Mettler-Toledo International Inc., Switzerland).

\subsection{Fabrication of caffeine loaded fish gelatin film and nanofibrous mats}

For the electrospinning, each dope solution was loaded into a syringe $(10 \mathrm{ml})$ fitted with a $22 \mathrm{G}$ needle and placed on a syringe pump (KD Scientific, USA) to precisely control the flow rate. The syringe needle was connected to a power supply (Chungpa EMT high voltage supply, Korea). A rolling stainless drum was electrically grounded and used as a collector. The flow rate of the dope solution was fixed at $0.3 \mathrm{ml} \mathrm{h}^{-1}$. Electrospinning was performed at room temperature, with a relative humidity $(\mathrm{RH})$ below $60 \%$. The distance from the tip to the collector was maintained at $15 \mathrm{~cm}$. The applied voltage was fixed at $15 \mathrm{kV}$. For the preparation of CAF-FG film, conventional film casting was performed with the CAF-FG solutions. $10 \mathrm{ml}$ of each solution was poured into a Petri dish and homogeneously spread on its surface. The CAF-FG solutions were left at $50{ }^{\circ} \mathrm{C}$ for $24 \mathrm{~h}$ allowing the water evaporation completely.

\subsection{Characterization}

The CAF crystallization of FG/CAF films were observed using bright field reflectance microscopy system (Eclipse LV100, Nikon, Japan). The morphology of the electrospun FG nanofiber mats was examined using field emission scanning electronic microscopy (FE-SEM) (AURIGA, Carl Zeiss, Germany), and the obtained images were analysed using image analysis software (Leopard, Korea). To investigate the flexibility of the CAF loaded FG drug carrier, the folding endurance test was performed. The folding endurance value was determined by repeatedly folding one film or nanofiber mat at same place till it breaks or folded up to 50 times. Attenuated total reflection Fourier transform infrared spectroscopy (ATR-FTIR, Thermo Scientific, Waltham, MA, USA) was used to identify the CAF incorporation into the FG nanofibers. The samples were examined within the wavenumber range of $2000-700 \mathrm{~cm}^{-1}$ and 32 scans with $8 \mathrm{~cm}^{-1}$ resolution were used to obtain the spectra. The X-ray diffraction analysis (XRD) was conducted using a Powder X-ray Diffractometer (D8 Advance, Bruker, Germany) with $\mathrm{Cu} \mathrm{K} \alpha$ radiation in a $2 \theta$ range of $5^{\circ}$ to $40^{\circ}$ at $40 \mathrm{mV}$ and $300 \mathrm{~mA}$. Differential scanning calorimetry (DSC) was carried out using a differential scanning calorimeter (DSC-Q1000, TA instrument, UK). Samples added into hermetically sealed pans and heated at $10{ }^{\circ} \mathrm{C}$ min $^{-1}$ from 30 to $240{ }^{\circ} \mathrm{C}$. The nitrogen gas flow rate was $40 \mathrm{ml} \mathrm{min}^{-1}$.

\subsection{Disintegration and wetting test}

The disintegration test was performed in $50 \mathrm{ml}$ of a dissolution medium. $0.1 \mathrm{~g}$ of CAF loaded FG film and nanofiber was soaked into the dissolution medium and disintegration behavior was 
recorded at 30 frames per second (I-phone 6, apple, USA) till the full disintegration occurred. The wettability of CAF loaded FG film and nanofibrous mats were measured by a procedure modified from the literature. ${ }^{15}$ The water-absorbable sponge was placed on a Petri dish with length of $5 \times 5 \mathrm{~cm}$. After the sponge was thoroughly wetted with distilled water $(10 \mathrm{ml})$, the excess water was completely drained out and a piece of CAF loaded FG film and nanofibrous mats were placed on the top of the wetted sponge. The wetting processes were recorded at 30 frames per second.

\subsection{In vitro caffeine release test}

For CAF release experiments, each sample was prepared with a weight of $0.50 \mathrm{~g}$. CAF-loaded FG films prepared by casting the spinning solutions and CAF powder were used as control samples for the release measurements. Each sample was immersed in $100 \mathrm{ml}$ of distilled water solution with a $100 \mathrm{rpm}$ stirring speed. One millilitre of supernatant was taken from the test solution at appropriate time intervals, and it was refilled with fresh water solution. The total amount of the drug in every sample of the electrospun membrane or casting film was determined after they had being immersed in distilled water for at least $1 \mathrm{~h}$ to reach the full dissolved state. Cumulative CAF release amount dissolved at specified time periods were plotted as the percentage released versus the time. Release amount of CAF was determined by HPLC (1260 Infinity, Agilent Technologies, California, USA) analysis. The mobile phase consisted of $20 \% \mathrm{v} / \mathrm{v}$ acetonitrile, $0.8 \% \mathrm{v} / \mathrm{v}$ trifluoroacetic acid, and $79.2 \% \mathrm{v} / \mathrm{v}$ distilled water. The analysis was carried out under isocratic conditions using a C18 column (ZORBAX Eclipse Plus C18, Agilent Technologies, California, USA). The column temperature was set to $40{ }^{\circ} \mathrm{C}$, and the flow rate of $1 \mathrm{ml} \mathrm{min}^{-1} .10 \mu \mathrm{l}$ of each sample was injected, and chromatograms were recorded at $276 \mathrm{~nm}$ for 4 min.

\section{Results and discussion}

\subsection{Characterization of caffeine-loaded fish gelatin nanofibers}

3.1.1. Morphology. The crystallization behavior of the drug during drug loading process is a major problem in the preparation of drug delivery carrier. ${ }^{23,24}$ Unexpected crystallization of the drug reduces drug loading efficiency and causes unexpected drug release behavior. Caffeine is a hydrophilic active ingredient with strong crystallization tendency. ${ }^{25}$ For this reason, we chose caffeine as the model drug. The maximum caffeine solubility in water at ambient room temperature is approximately $20 \mathrm{mg} \mathrm{ml}^{-1}(2.0 \%$, w/v). We consider this solubility of caffeine and prepared the various concentration of caffeine containing aqueous fish gelatin solution. The detailed compositions and properties of the caffeine loaded fish gelatin solutions are listed in Table 1 . To avoid confusion, we named the fish gelatin and caffeine as FG and CAF, respectively in the following context. The associated number refers the concentration (\%, w/v) of each sample. Fig. 1(a)-(f) shows the microscope images of CAF loaded FG films. As shown in the image of
Table 1 Characterization data of the dope solutions ${ }^{a}$

\begin{tabular}{llllll}
\hline Formulation & $\begin{array}{l}\text { Water } \\
(\mathrm{ml})\end{array}$ & $\begin{array}{l}\text { FG } \\
(\mathrm{g})\end{array}$ & $\begin{array}{l}\text { CAF } \\
(\mathrm{g})\end{array}$ & $\begin{array}{l}\text { Viscosity } \\
(\mathrm{Pa} \mathrm{s})\end{array}$ & $\begin{array}{l}\text { Conductivity } \\
\left(\mu \mathrm{S} \mathrm{m}^{-1}\right)\end{array}$ \\
\hline FG40/CAF0 & 10 & 4 & 0 & $0.582 \pm 0.02$ & $1061 \pm 35$ \\
FG40/CAF0.4 & 10 & 4 & 0.04 & $0.578 \pm 0.02$ & $1067 \pm 18$ \\
FG40/CAF0.8 & 10 & 4 & 0.08 & $0.608 \pm 0.03$ & $1066 \pm 24$ \\
FG40/CAF1.2 & 10 & 4 & 0.12 & $0.590 \pm 0.02$ & $1065 \pm 19$ \\
FG40/CAF1.6 & 10 & 4 & 0.16 & $0.606 \pm 0.07$ & $1065 \pm 20$ \\
FG40/CAF2.0 & 10 & 4 & 0.20 & $0.599 \pm 0.01$ & $1068 \pm 31$
\end{tabular}

${ }^{a} \mathrm{FG}=$ fish gelatin, $\mathrm{CAF}=$ caffeine, the number $x=x \%\left(\mathrm{~g} \mathrm{ml}^{-1}\right) \pm$ means the s.d. of the different observations $(N>3)$.

FG40/CAF0 and FG40/CAF0.4 film, there was no crystal structure during the film formation. In the case of FG40/CAF0.8 and FG40/CAF1.2, spherical crystalline nucleus were formed. The size of CAF crystal increased and fibrillation was observed with the CAF concentration increased up in the case of FG40/CAF1.6 and FG40/CAF2.0.

Electrospinning has been an alternative fabrication methodology to generate thin and flexible drug delivery systems. ${ }^{26}$ To determine the CAF addition effect on the morphology of the nanofiber, an analysis of SEM images was performed (Fig. 1(g)(1)). Formation of CAF loaded FG nanofibers without observable beads were successfully carried out via electrospinning process. The fibrous structure has the smooth surface, with no perceptible CAF crystals. It refers the CAF crystallization was restricted by fast solvent evaporation during the electrospinning compared to film casting. This fast and efficient water evaporation hinders the mobility of CAF molecules in the FG, thus favours the vitrified or solid solution state. ${ }^{27,28}$

The added amount of CAF into FG solution did not affect electrospinnability and the fiber morphology of FG nanofiber. In our previous study, the electrospinnability and resultant fiber diameter of FG were greatly affected by solution viscosity. However, there were no significant differences in the both of solution viscosity and electrical conductivity even though $20 \mathrm{mg} \mathrm{ml}^{-1}$ of CAF was introduced (Table 1). This indicates that the electrospinnability is not affected by the nature of the CAF, rather by the concentration of the FG solutions.

The folding endurance of FDDS carrier is an important mechanical property affected the packaging, handling, and storage. Fig. 2 shows the photo images of CAF loaded FG film and nanofiber before and after folding experiment. In the case of CAF loaded FG film after 5 times of folding process induce the crack of film and finally broken to separated parts. To give the mechanical flexibility, many plasticizers such as glycerol, propylene glycol, and sorbitol have been widely used. ${ }^{29,30}$ However, CAF loaded FG nanofiber had passed the 50 times of folding process without any crack or broken pieces. It indicated the FG nanofibrous mat has flexibility compared to film forms due to inter porous network of nanofibers.

3.1.2. Physical status and compatibility of caffeine and fish gelatin. To find out the incorporation of CAF into FG nanofibers, ATR-FTIR analysis was performed and the results was 

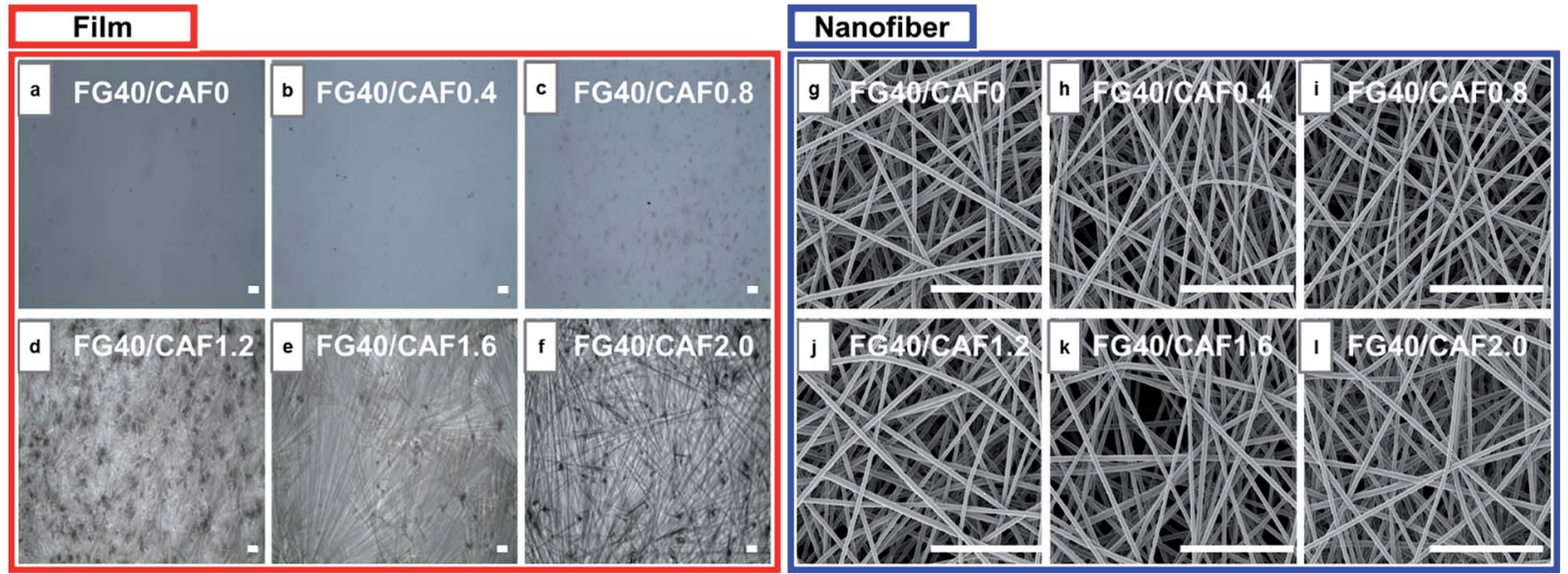

Fig. 1 (a-f) Brightfield reflectance microscope images of increasing concentrations of caffeine loaded fish gelatin film. ( $\mathrm{g}-\mathrm{l}$ ) Field emission scanning microscope (FE-SEM) images of increasing concentrations of electrospun caffeine loaded fish gelatin nanofiber. Caffeine loading ranges from $0-2 \%$. Scale bar represents $5 \mu \mathrm{m}$.

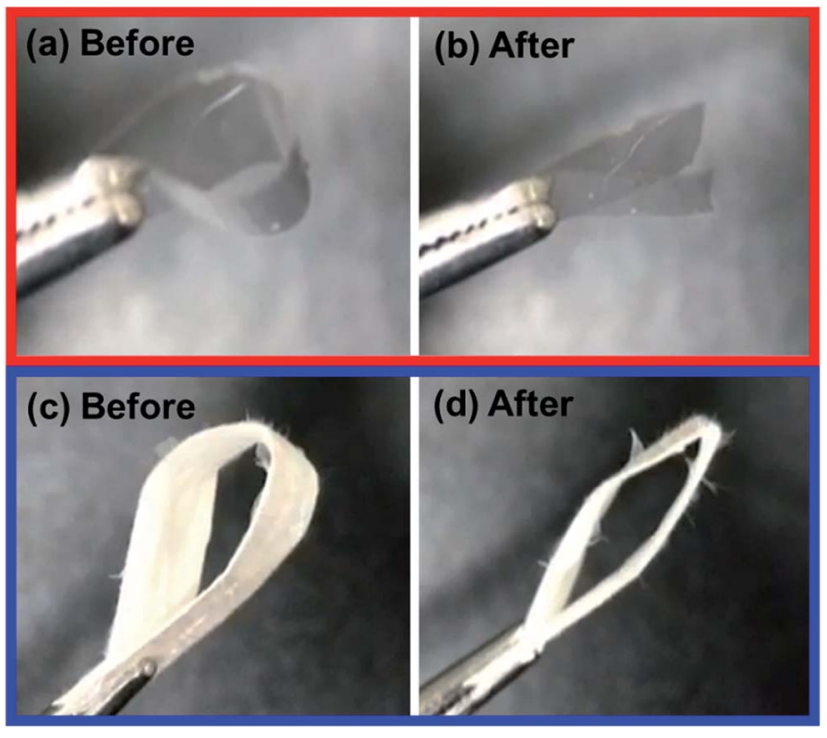

Fig. 2 Photographies of caffeine loaded fish gelatin film (a), (b) and nanofiber (c), (d) before and after the folding endurance test respectively.

shown in Fig. 3(a). The pure CAF has the characteristic peaks at $1700 \mathrm{~cm}^{-1}$ (C=N stretching), $1285 \mathrm{~cm}^{-1}$ (C-N stretching), $973 \mathrm{~cm}^{-1}\left(\mathrm{~N}-\mathrm{CH}_{3}\right.$ stretching) and $740 \mathrm{~cm}^{-1}(\mathrm{O}=\mathrm{C}-\mathrm{C}$ deformation), respectively. ${ }^{31}$ The pure FG revealed characteristic absorption bands at $1640-1650 \mathrm{~cm}^{-1}(\mathrm{C}=\mathrm{O}$ stretching of amide $\mathrm{I})$ and $1540-1550 \mathrm{~cm}^{-1}\left(\mathrm{~N}-\mathrm{H}\right.$ bending of amide II), and $1240-1250 \mathrm{~cm}^{-1}$ (N-H bending of amide III), respectively. ${ }^{32,33}$ The electrospun CAF loaded FG film and nanofibrous mat showed all of the characteristic peaks of each of CAF and FG and the absorbance of CAF was decreased due to the strong intensity of FG nanofiber itself.

To determine the physical state of CAF in the FG film and nanofiber, X-ray diffraction (XRD) analysis was carried out and its results are shown in Fig. 3(b). Powder form of CAF has an ordered crystalline lattice which depicts the characteristic high intensity peaks in the X-ray diffraction pattern. The characteristic diffraction peaks of CAF powder appeared at diffraction angles 2 thetas of $11.6^{\circ}, 11.9^{\circ}, 26.4^{\circ}$ and $27.8^{\circ}$. Otherwise, the diffraction patterns of FG exhibit a diffuse background pattern with a diffraction halos, implies the amorphous state of the FG polymer. In the case of FG40/CAF2.0 film, the high intensity of CAF crystal peaks at $11.9^{\circ}$ was observed. It indicated that the CAF crystallization occurred during the film formation which is consistent with microscope image results. In the case of FG40/CAF2.0 nanofiber, all the CAF peaks were absent which indicates that the CAF is incorporated in the FG fibers with amorphous physical form. Differential scanning calorimetry (DSC) analysis results also support the prevention of CAF crystallization during the electrospinning process (Fig. 3(c)). The DSC curve of pure CAF exhibited a single endothermic peak at 235.5 ${ }^{\circ} \mathrm{C}$ which corresponding to crystalline $\mathrm{CAF}$ melting point. ${ }^{34,35}$ As amorphous polymers, FG did not show any fusion peaks or phase transitions. In the case of CAF loaded FG film, there was an endothermic peak of crystal CAF melting peak which indicates the crystallization during the slow water evaporation process. ${ }^{36}$ On the other hand, the DSC thermograms of the CAF loaded FG nanofiber did not show the characteristic melting peak of crystalline CAF. This finding suggests that the crystalline CAF was converted into the amorphous state during the dissolving into water. The electrospinning process prevents the crystallization and remains the amorphous solid solution state of CAF which is well incorporated into the FG nanofiber.

3.1.3. Disintegration and wetting behavior of caffeine loaded fish gelatin nanofiber. The disintegration and wetting behavior of the drug delivery polymer carrier are the most important factors in the FDDS systems. It is affected by the hydrophilic nature of polymer, porosity and surface area of materials. ${ }^{37,38}$ The disintegration profiles of CAF loaded FG drug delivery carrier from different fabrication method are shown in Fig. 4. As shown in the figure, the electrospun FG nanofiber has 

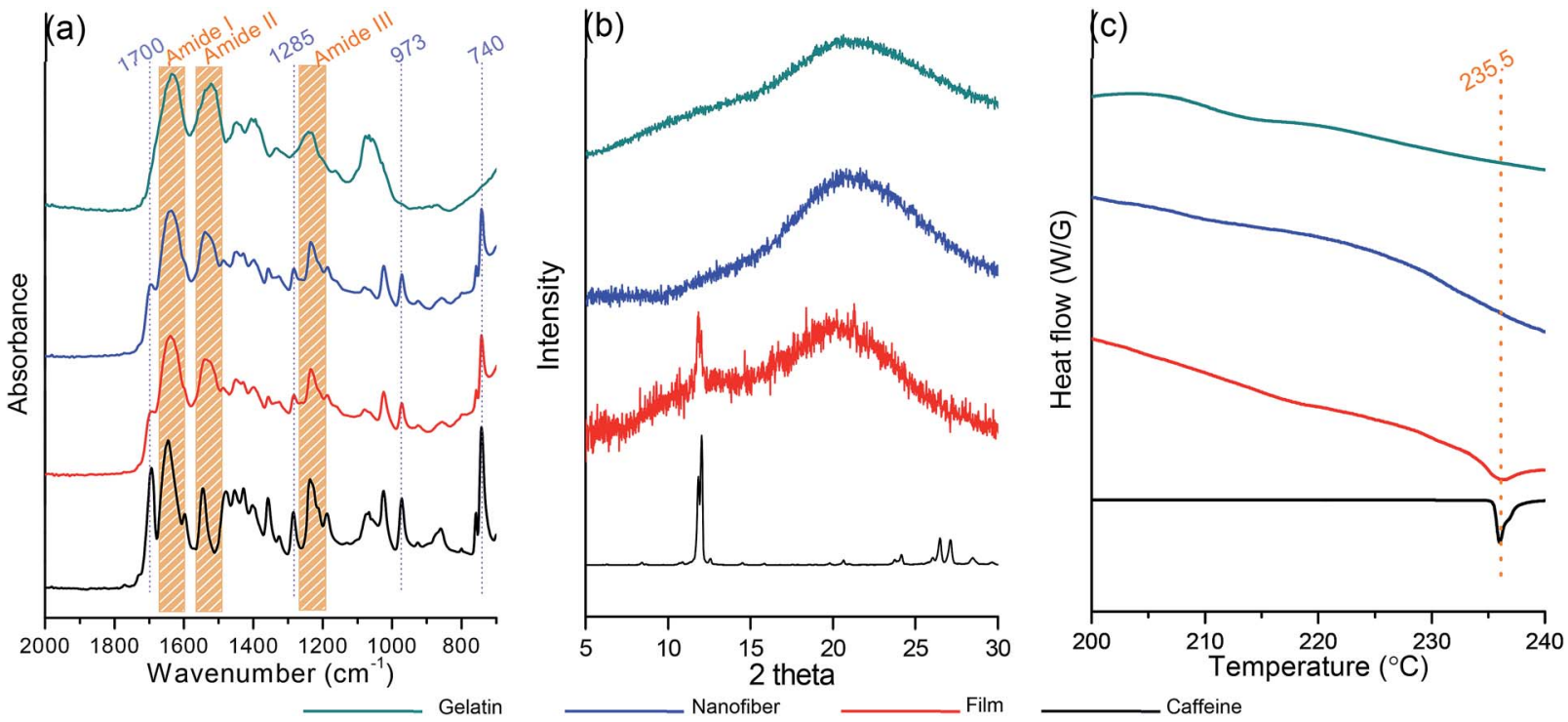

Fig. 3 (a) ATR-FTIR spectra, (b) XRD patterns, and (c) DSC thermograms of powdered fish gelatin (green) and caffeine (black), fish gelatin film added with caffeine $20 \mathrm{mg} \mathrm{ml}^{-1}$ (red, FG40/CAF2.0), fish gelatin nanofiber added with caffeine $20 \mathrm{mg} \mathrm{ml}^{-1}$ (blue, FG40/CAF2.0).

significant fast dissolution behavior compared to its film form. The total disintegration time of each FG nanofiber and film are approximately 1.5 and $40 \mathrm{~s}$ respectively. It is due to the high surface to volume ratio and huge matrix surface area of the nanofibrous structure.

The main target of FDDS is not only the general patients who need rapid drug delivery, such as pain relievers but also patients who is unavailable to swallow or absorb water. For this reason, wetting behavior in the small amount of saliva condition is very important. The wetting experiment was carried out using the small amount water $(10 \mathrm{ml})$ absorbed sponge material which considers the minute volumes of saliva.

Fig. 5 shows the photographs of the wetting process of FG40/ CAF2.0 films and nanofiber. Although the wetting time of each

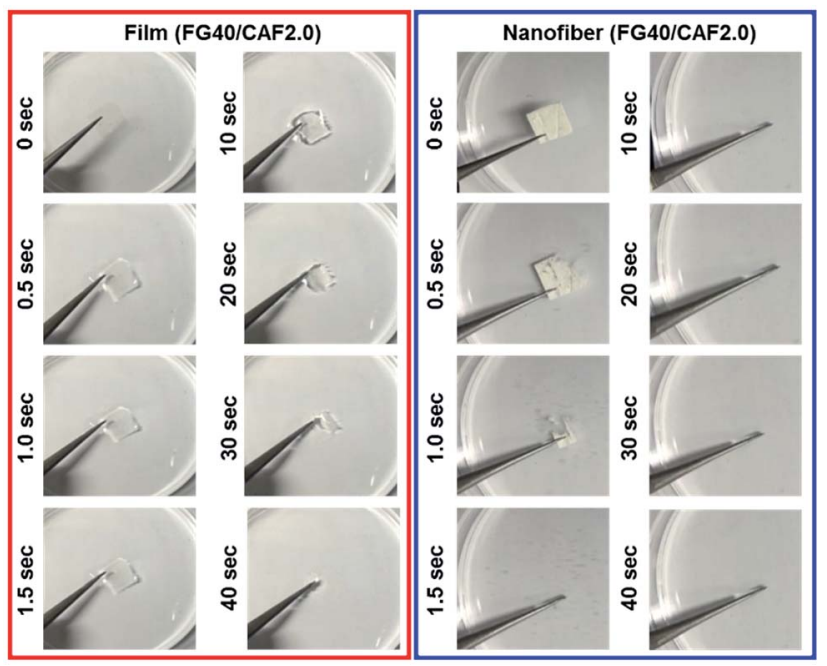

Fig. 4 Dissolution behavior of fish gelatin film added with caffeine $20 \mathrm{mg} \mathrm{ml}^{-1}$ (red, FG40/CAF2.0), fish gelatin nanofiber added with caffeine $20 \mathrm{mg} \mathrm{ml}^{-1}$ (blue, FG40/CAF2.0). sample increased compared to disintegration time, the nanofiber quickly absorbed water from the wet sponge and finally could be disintegrated within $5 \mathrm{~s}$. However, when FG40/CAF2.0 films absorbed water, they converted to transparent gels, with the gel state maintained up to 3 minutes because the amount of water was not enough to disintegrate the film. This indicated that the nanofibrous FG is an ideal formulation for the FDDS carrier within the presence of the minute volume of saliva condition.

\subsection{In vitro caffeine release behavior}

The drug release behavior is affected not only the physicochemical properties of the carrier polymer but also the physical

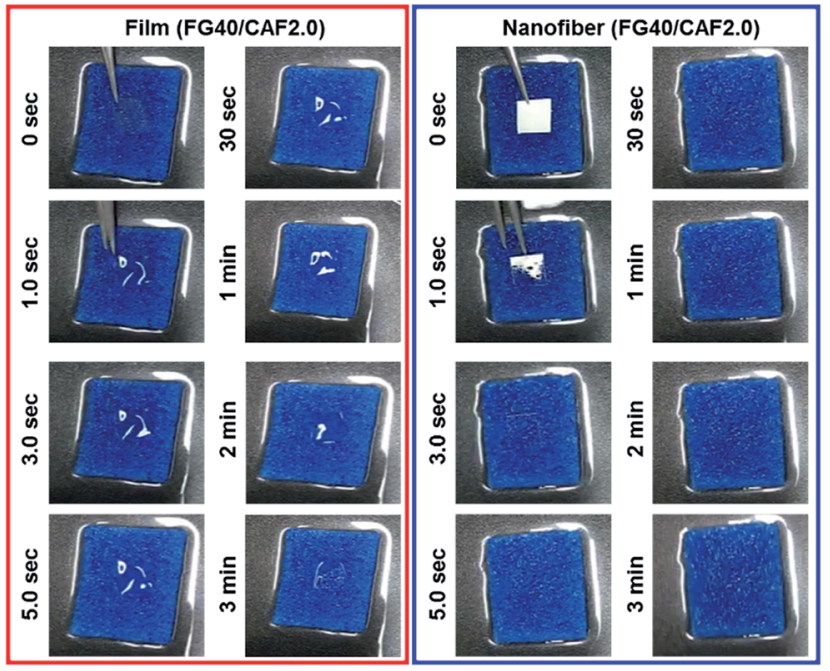

Fig. 5 Wetting behavior of fish gelatin film added with caffeine $20 \mathrm{mg} \mathrm{ml}^{-1}$ (red, FG40/CAF2.0), fish gelatin nanofiber added with caffeine $20 \mathrm{mg} \mathrm{ml}^{-1}$ (blue, FG40/CAF2.0). 

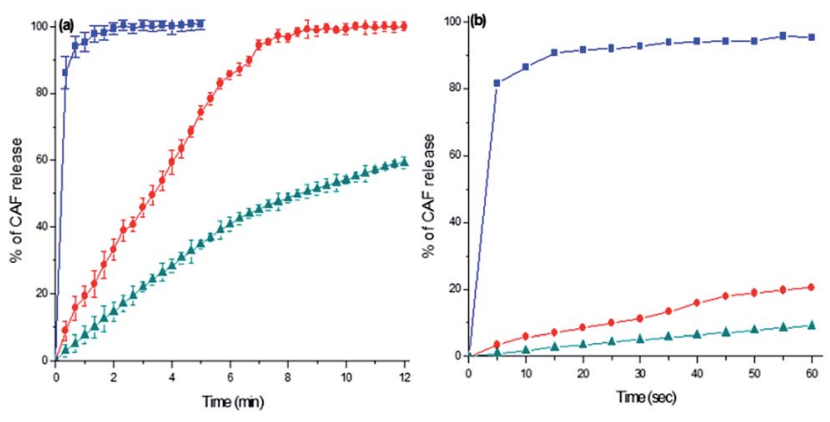

Fig. 6 In vitro caffeine release behavior of caffeine loaded fish gelatin nanofiber (blue square, FG40/CAF2.0), film (red circle, FG40/CAF2.0), and free caffeine powder (green triangle, $0.20 \mathrm{mg} \mathrm{ml}^{-1}$ ) (a). Initial caffeine release behavior of 1 min time period (b). The error bars represent the s.d. of the different observations $(N>4)$.

state of the loaded drug. For the purpose of comparing the in vitro $\mathrm{CAF}$ release behavior, three different $\mathrm{CAF}$ forms were prepared-powdered CAF, CAF loaded FG film, and CAF incorporated FG nanofibrous membrane. The in vitro CAF release experiment results are shown in Fig. 6. The CAF release rate of film and nanofibrous samples was considerably faster than that of free powdered CAF. These fast CAF release rate could be attributed to the hydrophilicity of FG. Comparing the two FG based CAF carriers of film and nanofiber, fine nanofibrous carrier showed the ultrafast CAF release rate than that of film forms. FG nanofiber took within $10 \mathrm{~s}$ to release more than $90 \%$ of CAF contentment and most of CAF was diffused out to bulk solutions completely within 1 minute. In the case of FG film, the initial release rate of CAF was slower than FG nanofiber and the complete release of CAF took within 7 minutes. Such a high enhancement of fast CAF release behavior of FG nanofiber can be explained by higher dissolution medium accessibility of the FG nanofiber and the amorphous state CAF present in FG nanofiber. ${ }^{39}$ The higher surface to volume ratio provided that the dissolving medium can be reached fast to FG nanofiber matrix. $^{40}$ After that, the amorphous CAF dispersed in FG nanofiber makes ultrafast CAF release compare to the crystalline state of CAF.

\section{Conclusions}

In this study, we successfully fabricated the CAF loaded FG nanofibrous membrane as FDDS carrier. The electrospun CAF loaded FG nanofiber had the fastest integration rate and $\mathrm{CAF}$ release behavior which can be attributed to the following reasons: (1) FG has hydrophilic properties and it can be easily electrospun with $200 \mathrm{~nm}$ of average fiber diameter using water as a solvent without any organic solvent or polymer additive. The three-dimensional continuous web structure of the nanofibrous membrane provided a large surface to volume ratio to absorb and disintegrate fast by water molecules. This nanofibrous membrane also had a higher porosity for the water molecules to diffuse and contact with the inner parts of FG membrane. (2) The fast water evaporation during electrospinning and interaction between FG and CAF prevented the crystallization of CAF itself; thus, the CAF molecules can dissolve and release spontaneously with the disintegration of FG nanofiber.

Comparing the results obtained above this study and the previous polymer nanofiber-based FDDS carriers, we could find that the electrospun nanofiber using the aqueous solution of FG has the following advantages. Table 2 shows the characteristics of the caffeine-loaded polymeric FDDS nanofibrous carrier reported up to now. First, electrospinning of aqueous FG is environmentally friendly and economical because it uses water as a solvent and electrospinning process is accomplished in an ambient environment condition. Furthermore, the aqueous solvent system could minimize safety problems that may occur when using organic solvents. ${ }^{41}$ Secondly, the FG electrospinning can carry caffeine in an amount close to the maximum solubility in water while preventing crystallization. Third, FG electrospinning can produce nanofibers with the thinnest diameter of $200 \mathrm{~nm}$ on average. Finally, CAF loaded FG nanofibers have the fastest CAF release rate than other polymer-based nanofibers.

This is the first report using aqueous FG natural biopolymer based nanofiber as a FDDS formulation and the electrospun FG nanofiber can be used in biomedical fields especially for the ultrafast delivery of the hydrophilic drug or active ingredient.

\section{Conflicts of interest}

There are no conflicts to declare.

\section{Acknowledgements}

We thank Dr Chris Holland (The University of Sheffield) for discussion of the experimental results and proofreading the manuscript. This work was supported by the Technology Innovation Program (10076478, Development of natural protein

Table 2 Characterization data of polymeric nanofibrous caffeine delivery system. Figures in bold represent best reported properties to date

\begin{tabular}{llllll}
\hline Polymer & Solvent & $\begin{array}{l}\text { Drug loading } \\
\text { contents }(\%, \mathrm{w} / \mathrm{v})\end{array}$ & $\begin{array}{l}\text { Fiber diameter } \\
(\mathrm{nm})\end{array}$ & $\begin{array}{l}\text { Total caffeine } \\
\text { release time }(\mathrm{s})\end{array}$ & $\begin{array}{l}\text { Caffeine release } \\
\text { rate }(\% / \mathrm{s})\end{array}$ \\
\hline PCL & TFE : DMF $(90: 10 \mathrm{v} / \mathrm{v})$ & $0.88-1.32$ (calculated) & $500-600$ & 900 & 0.00098 \\
PVA & Water : acetic acid $(1: 1 \mathrm{v} / \mathrm{v})$ & 0.11 (calculated) & $600-700$ & 900 & 9 \\
PVA & Distilled water & 1.00 & $260-370$ & 60 & 0.0015 \\
PVP & Ethanol & 0.65 & $443-1453$ & $60-100$ & 0.0167 \\
Fish gelatin & Distilled water & $\mathbf{2 . 0 0}$ & $\mathbf{2 0 0 - 2 2 0}$ & $\mathbf{2 0}$ & $0.006-0.010$ \\
\end{tabular}


composite fibers and materials for skin care products having improved moisturizing ability and mechanical properties) funded By the Ministry of Trade, Industry \& Energy (MOTIE, Korea).

\section{Notes and references}

1 S. V. Sastry, J. R. Nyshadham and J. A. Fix, Pharm. Sci. Technol. Today, 2000, 3, 138-145.

2 F. Cilurzo, I. E. Cupone, P. Minghetti, F. Selmin and L. Montanari, Eur. J. Pharm. Biopharm., 2008, 70, 895-900.

3 A. Chaturvedi, P. Srivastava, S. Yadav, M. Bansal, G. Garg and P. K. Sharma, Curr. Drug Delivery, 2011, 8, 373-380.

4 A. F. Borges, C. Silva, J. F. J. Coelho and S. Simões, J. Controlled Release, 2015, 206, 1-19.

5 H. Kathpalia and A. Gupte, Curr. Drug Delivery, 2013, 10, 667684.

6 S. Bahrainian, M. Abbaspour, K. Maryam and P. T. Moghadam, Jundishapur J. Nat. Pharm. Prod., 2016, DOI: $10.17795 /$ jjnpp-34267.

7 V. A. Perumal, T. Govender, D. Lutchman and I. Mackraj, Drug Dev. Ind. Pharm., 2008, 34, 1036-1047.

8 B. Y. Shekunov and P. York, J. Cryst. Growth, 2000, 211, 122136.

9 S. Seif, L. Franzen and M. Windbergs, Int. J. Pharm., 2015, 478, 390-397.

10 K. Garg and G. L. Bowlin, Biomicrofluidics, 2011, 5, 013403.

11 F. Sharifi, A. C. Sooriyarachchi, H. Altural, R. Montazami, M. N. Rylander and N. Hashemi, ACS Biomater. Sci. Eng., 2016, 2, 1411-1431.

12 F. L. Lopez, G. C. Shearman, S. Gaisford and G. R. Williams, Mol. Pharmaceutics, 2014, 11, 4327-4338.

13 G. Verreck, I. Chun, J. Peeters, J. Rosenblatt and M. E. Brewster, Pharm. Res., 2003, 20, 810-817.

14 X. Li, M. A. Kanjwal, L. Lin and I. S. Chronakis, Colloids Surf., $B, 2013,103,182-188$.

15 P. R. Vuddanda, A. P. Mathew and S. Velaga, React. Funct. Polym., 2016, 99, 65-72.

16 D.-G. Yu, X.-X. Shen, C. Branford-White, K. White, L.-M. Zhu and S. W. A. Bligh, Nanotechnology, 2009, 20, 055104.

17 J. C. Bragg, H. Kweon, Y. Jo, K. G. Lee and C.-C. Lin, RSC Adv., 2016, 6, 114353-114360.

18 M. J. Dille, M. N. Hattrem and K. I. Draget, Food Hydrocolloids, 2017, DOI: 10.1016/j.foodhyd.2016.12.028.

19 Y. Piao and B. Chen, RSC Adv., 2016, 6, 6171-6181.

20 A. d. T. Alfaro, E. Balbinot, C. I. Weber, I. B. Tonial and A. Machado-Lunkes, Food Eng. Rev., 2014, 7, 33-44.

21 G. Boran and J. M. Regenstein, Adv. Food Nutr. Res., 2010, 60, 119-143.
22 H. W. Kwak, M. Shin, J. Y. Lee, H. Yun, D. W. Song, Y. Yang, B.-S. Shin, Y. H. Park and K. H. Lee, Int. J. Biol. Macromol., 2017, 102, 1092-1103.

23 J. Hadgraft and M. E. Lane, Expert Opin. Drug Delivery, 2016, 13, 817-830.

24 P. Jain and A. K. Banga, Int. J. Pharm., 2010, 394, 68-74.

25 Nonappa and E. Kolehmainen, Gels, 2016, $2,9$.

26 M. Afshari, Electrospun Nanofibers, Woodhead Publishing, Cambridge, 2016.

27 Z. K. Nagy, A. Balogh, B. Démuth, H. Pataki, T. Vigh, B. Szabó, K. Molnár, B. T. Schmidt, P. Horák, G. Marosi, G. Verreck, I. Van Assche and M. E. Brewster, Int. J. Pharm., 2015, 480, 137-142.

28 G. Verreck, I. Chun, J. Rosenblatt, J. Peeters, A. V. Dijck, J. Mensch, M. Noppe and M. E. Brewster, J. Controlled Release, 2003, 92, 349-360.

29 M. Thomazine, R. A. Carvalho and P. J. A. Sobral, J. Food Sci., 2005, 70, E172-E176.

30 F. M. Vanin, P. J. A. Sobral, F. C. Menegalli, R. A. Carvalho and A. M. Q. B. Habitante, Food Hydrocolloids, 2005, 19, 899-907.

31 M. Baranska and H. Schulz, in The Alkaloids: Chemistry and Biology, ed. G. A. Cordell, Academic Press, Cambridge, 1st edn, 2009, ch. 4, vol. 67, pp. 217-255.

32 S. Kim, M. E. Nimni, Z. Yang and B. Han, J. Biomed. Mater. Res., Part B, 2005, 75, 442-450.

33 J. Xu, N. Cai, W. Xu, Y. Xue, Z. Wang, Q. Dai and F. Yu, Nanotechnology, 2013, 24, 025701.

34 K. Klímová and J. Leitner, Thermochim. Acta, 2012, 550, 5964.

35 S. S. Pinto and H. P. Diogo, J. Chem. Thermodyn., 2006, 38, 1515-1522.

36 J. X. Wu, M. Yang, F. van den Berg, J. Pajander, T. Rades and J. Rantanen, Eur. J. Pharm. Sci., 2011, 44, 610-620.

37 G. Bruni, L. Maggi, L. Tammaro, R. Di Lorenzo, V. Friuli, S. D'Aniello, M. Maietta, V. Berbenni, C. Milanese, A. Girella and A. Marini, Int. J. Pharm., 2016, 511, 190197.

38 D.-G. Yu, C. Branford-White, K. White, X.-L. Li and L.-M. Zhu, AAPS PharmSciTech, 2010, 11, 809-817.

39 E. Karavas, E. Georgarakis, M. P. Sigalas, K. Avgoustakis and D. Bikiaris, Eur. J. Pharm. Biopharm., 2007, 66, 334-347.

40 F. Ding, H. Deng, Y. Du, X. Shi and Q. Wang, Nanoscale, 2014, 6, 9477-9493.

41 Y. Kishimoto, H. Morikawa, S. Yamanaka and Y. Tamada, Mater. Sci. Eng., C, 2017, 73, 498-506. 